\title{
Reduction of costs for anemia-management drugs associated with the use of ferric citrate
}

This article was published in the following Dove Press journal: International Journal of Nephrology and Renovascular Disease 20 May 2014

Number of times this article has been viewed

\author{
Anila Thomas' \\ Leif E Peterson² \\ 'Clinical Pharmacy Services, Houston \\ Methodist Hospital, Houston, TX, \\ USA; ${ }^{2}$ Center for Biostatistics, \\ Houston Methodist Research \\ Institute, Houston, TX, USA
}

Background: Ferric citrate is a novel phosphate binder which has the potential to reduce usage of erythropoietin-stimulating agents (ESAs) and intravenous (IV) iron used for anemia management during hemodialysis (HD) among patients with end-stage renal disease (ESRD). Currently, the potential health care cost savings on a national scale due to the use of ferric citrate in ESRD are undetermined.

Methods: Per-patient-per-year costs of ESAs (Epogen ${ }^{\circledR}$ and Aranesp ${ }^{\circledR}$ [Amgen Inc., CA, USA]) and IV iron (Venofer ${ }^{\mathbb{R}}$ [American Regent, Inc., NY, USA] and Ferrlecit ${ }^{\mathbb{R}}$ [Sanofi US, Bridgewater, NJ, USA]) were based on RED BOOK ${ }^{\mathrm{TM}}$ (Truven Health Analytics New York, NY, USA) costs combined with the Centers for Medicare and Medicaid Services (CMS) base rate and actual usage in 2011 for the four drugs. The annual number of outpatients undergoing HD in the US was based on frequencies reported by the USRDS (United States Renal Data System). Monte Carlo uncertainty analysis was performed to determine total annual costs and cost reduction based on ferric citrate usage.

Results: Total annual cost of ESAs and IV iron for anemia management in ESRD determined by Monte Carlo analysis assuming CMS base rate value was 5.127 (3.664-6.260) billion USD. For actual utilization in 2011, total annual cost of ESAs and IV iron was 3.981 (2.780-4.930) billion USD. If ferric citrate usage reduced ESA utilization by $20 \%$ and IV iron by $40 \%$, then total cost would be reduced by $21.2 \%$ to $4.038(2.868-4.914)$ billion USD for the CMS base rate, and by $21.8 \%$ to 3.111 (2.148-3.845) billion USD, based on 2011 actual utilization.

Conclusion: It is likely that US health care costs for anemia-management drugs associated with ESRD among HD patients can be reduced by using ferric citrate as a phosphate binder.

Keywords: end-stage renal disease, hemodialysis, erythropoietin-stimulating agents, supplemental iron, phosphate binder, Monte Carlo analysis

\section{Introduction}

In 2011, the prevalence in the US of end-stage renal disease (ESRD) was 507,326, and there were 88,931 deaths attributable to ESRD. ${ }^{1}$ Medicare costs for ESRD were $\$ 34.3$ billion in 2011, accounting for over $6 \%$ of the total Medicare budget. On January 1, 2011, Medicare enacted an ESRD bundled prospective payment system for reimbursing dialysis units. Through this payment bundle, Medicare and beneficiaries paid approximately US\$10 billion annually for dialysis services, 25\% of which was due to medications used for the management of anemia. ${ }^{2}$ In 2010, erythropoietin-alfa for the management of anemia in ESRD was the highest-expenditure drug covered by Medicare Part B, accounting for US\$2 billion in Medicare expenditure. ${ }^{3}$ The most commonly prescribed medications for the management of anemia in ESRD patients are the erythropoietin-stimulating agents (ESAs; epoetin-alfa and darbepoetin-alfa)
Correspondence: Leif E Peterson Center for Biostatistics, Houston Methodist Research Institute, 6565 Fannin Street, Suite MGJ6-03I, Houston, TX 77030, USA Email lepeterson@houstonmethodist.org (c) (i) (5) 2014 Thomas and Peterson. This work is published by Dove Medical Press Limited, and licensed under Creative Commons Attribution - Non Commercial (unported, v3.0) License. The full terms of the license are available at http:///creativecommons.org/licenses/by-n/3.0/. Non-commercial uses of the work are permitted without any further ins beyond the scope of the License are administered by Dove Medical Press Limited. Information on how to request permission may be found at: http://www.dovepress.com/permissions.php 
and intravenous (IV) iron supplements (iron sucrose and sodium ferric gluconate complex).

Erythropoietin produced by the kidneys stimulates bone marrow production of erythroid progenitor cells, which give rise to heme-iron carrying erythrocytes. ${ }^{4}$ As kidney function declines, renal production of erythropoietin also declines, and the prevalence of anemia increases. Consequently, ESRD patients require recombinant ESAs in order to maintain adequate hemoglobin levels. ${ }^{5-7}$ Iron plays a crucial role in the synthesis of heme and is embedded in the porphyrin rings of the hemoglobin molecule. Patients with advanced stages of renal disease are susceptible to iron deficiency, primarily due to inadequate gastrointestinal absorption of iron, blood loss, chronic inflammation, and iron utilization for erythropoiesis. ${ }^{8}$ As a result of this iron deficiency, IV iron supplementation is administered to maintain adequate hemoglobin levels.

The International Society of Nephrology's guidelines for the management of anemia in chronic kidney disease recommend the use of ESAs and iron supplements, albeit judiciously. ${ }^{9}$ For patients on dialysis, initiation of ESA therapy may be considered when the hemoglobin level is between 9 and $10 \mathrm{~g} / \mathrm{dL}$. Undesired consequences of ESA therapy include increased potential for thromboembolism and cardiovascular events, and high ESA drug acquisition costs. Initiation of iron supplements in the management of anemia can reduce both the dose of ESAs required and the need for blood transfusions. Bone mineral disorders in advanced kidney disease also lead to hyperphosphatemia, vascular calcification, and increased mortality. ${ }^{10,11}$ Phosphate binders are therefore administered to dialysis patients to reduce serum phosphate levels, thereby reducing the severity of hyperphosphatemia.

In light of the multiple sequelae of ESRD requiring the use of phosphate binders, ESAs, and IV iron, strategies are currently under development to minimize ESA utilization, reduce national costs, decrease pill burden, and manage anemia and concurrent disease states in dialysis patients. One such strategy is the development of ferric citrate coordination complex (Zenerex ${ }^{\mathrm{TM}}$, Keryx Biopharmaceuticals, Inc., New York, NY, USA). Ferric citrate is a novel phosphate binder which also has the potential to reduce requirements for ESAs and IV iron supplementation. A Phase III study of $\mathrm{n}=183$ ESRD patients compared ferric citrate to comparator groups in two phases: the first phase which compared ferric citrate to sevelamer or calcium acetate and the second phase which compared it with placebo (Unpublished, Keryx Biopharmaceuticals; available from http:www.Keryx.com). Patients treated with ferric citrate showed a phosphorus reduction from 5.2 to $4.9 \mathrm{mg} / \mathrm{dL}$ over 4 weeks, compared with an increase in the placebo-controlled comparator group from 5.3 to $7.2 \mathrm{mg} / \mathrm{dL}(P<0.0001)$. Transferrin saturation increased in the ferric citrate group from $31 \%$ to $39 \%$ over 52 weeks, while it remained largely unchanged in the comparator group, which used either sevelamer or calcium acetate as phosphate binders $(P<0.0001)$. Ferric citrate use resulted in reduced IV iron usage by a median of $51.6 \%$ and reduced ESA usage by a median of $27.1 \%$ over 52 weeks. Decline in hemoglobin level was lower in the ferric citrate arm $(-0.2$ versus $-0.6, P=0.01)$. A Phase II randomized, placebocontrolled, double-blind, multicenter study of ESRD patients receiving ferric citrate $3 \mathrm{~g} /$ day for 4 weeks demonstrated a change in serum phosphorus of $-2.16 \mathrm{mg} / \mathrm{dL}$, serum iron of $26.6 \mathrm{mg} / \mathrm{dL}$, ferritin of $15.55 \mathrm{ng} / \mathrm{mL}$, transferrin saturation of $8.19 \%$, and hemoglobin of $0.56 \mathrm{~g} / \mathrm{dL} .{ }^{12}$ Adverse effects in both studies were similar between groups and were gastrointestinal in nature, with no overall serious adverse events. A meta-analysis of three clinical studies found a mean increase from baseline in serum ferritin of $44.98 \mathrm{ng} / \mathrm{mL}$ and mean transferrin saturation of $2.23 \%$ among $n=265$ patients treated with ferric citrate for 4 weeks. ${ }^{13}$ A study of cost savings associated with 2 months of ferric citrate use found reductions in ESA dose of 500 units and in iron dose of $5.79 \mathrm{mg}$ per dialysis session. ${ }^{14}$ Based on the findings of these studies, ferric citrate has the potential to reduce costs of ESAs and IV iron by increasing iron and ferritin levels, thereby reducing ESAs and IV iron dose requirements.

Ferric citrate is approved for anemia management in ESRD in Japan and is expected to be approved by the US Food and Drug Administration (FDA) on June 7, 2014 as an oral treatment for hyperphosphatemia in chronic kidney disease. A cost-offset study from a managed-care perspective by Mutell et al demonstrated the potential for cost savings with the use of ferric citrate compared with other phosphate binders. In their study, monthly ESA cost was projected to be reduced by $8.15 \%$, and IV iron cost by $33.2 \% .{ }^{15} \mathrm{~A}$ Monte Carlo simulation demonstrated a reduction of US\$160 per month in overall dialysis cost per patient with the use of ferric citrate. Currently, the total potential health care cost savings on a national scale due to the use of ferric citrate in ESRD are undetermined.

The purpose of this investigation was to model the projected US total annual costs of ESAs and IV iron for ESRD, and the cost reduction for ESAs and iron for ESRD, based on the use of ferric citrate as a phosphate binder agent. We calculated per-patient-per-year (PPPY) costs of ESAs and iron using RED BOOK ${ }^{\mathrm{TM}}$ (Truven Health Analytics New York, NY, USA) values adjusted for the Centers for Medicare and Medicaid Services (CMS) base rate utilization and actual 2011 utilization reported by CMS. Monte Carlo uncertainty 
analysis was employed to obtain median total annual cost of ESAs and iron, and projected cost savings of ESAs and iron when ferric citrate is used as a binder.

\section{Methods}

\section{ESA and IV iron costs}

A recent report published by CMS on costs of anemiamanagement drugs for hemodialysis (HD) patients provided mean per HD session doses for the ESA drugs (Epogen ${ }^{\circledR}$ and Aranesp ${ }^{\circledR}$ [Amgen Inc., CA, USA]) and IV iron drugs (Venofer $^{\circledR}$ [American Regent, Inc., NY, USA] and Ferrlecit ${ }^{\circledR}$, [Sanofi US, Bridgewater, NJ, USA]). ${ }^{2}$ Utilization costs of these four drugs were based on RED BOOK costs combined with CMS base rate and actual usage in 2011. Regarding ESAs and CMS base rates, Epogen was used much more frequently than Aranesp, with a calculated PPPY cost of US\$12,243. For Aranesp, the calculated PPPY cost was US\$1,426. For actual 2011 utilization, the PPPY costs for Epogen and Aranesp were US\$9,545 and US\$543. For iron, when applying the CMS base rate to RED BOOK costs, Venofer was used more frequently than Ferrlecit, and had a calculated PPPY cost of US\$1,057. On the other hand, Ferrlecit usage resulted in a calculated PPPY cost of US\$432. Calculated PPPY costs for Venofer and Ferrlecit based on actual 2011 utilization were US\$1,148 and US\$209, respectively. Table 1 lists the sources of information used for determining utilization costs, and the resulting lower, middle, and upper cost boundaries. We set the upper bound of triangle distributions equal to the mode plus $10 \%$.

\section{Monte Carlo uncertainty analysis}

Monte Carlo analysis was employed for folding together 15,000 random draws of quantiles from probability distributions simulated to represent uncertainty of the various inputs. The annual total cost model was based on the relationship

$$
y_{\text {cost }}=\left(x_{\mathrm{ESA}}+x_{\mathrm{Fe}}\right) \times x_{\# \mathrm{HD}}
$$

where $y_{\text {cost }}$ is the projected annual cost determined from the sum of randomly sampled quantiles ( $x$-values) of ESA and IV iron PPPY costs, multiplied by a random quantile for the number of patients undergoing HD per year (see Table 1 for quantile $x$ notation). The total cost model was only used twice: once for the total annual cost based on the CMS base rate, and once for the total annual cost based on the 2011 actual utilization reported by CMS. Likewise, the annual cost-reduction model determined the fraction of PPPY ESA and IV iron cost saved by applying reduction factors which represented the assumed proportional reduction in ESA and IV iron usage as a result of using ferric citrate. The cost-reduction model for ferric citrate usage was functionally composed as

$$
y_{\text {reduction }}=\left(x_{\mathrm{ESA}} \times x_{\mathrm{RF} 1}+x_{\mathrm{Fe}} \times x_{\mathrm{RF} 2}\right) \times x_{\mathrm{HHD}} \text {, }
$$

\begin{tabular}{|c|c|c|c|c|}
\hline Source & Description & Value & Distribution $^{a}$ & $\begin{array}{l}\text { Monte Carlo } \\
\text { quantile }\end{array}$ \\
\hline USRDS' & $\begin{array}{l}\text { Number of HD patients per } \\
\text { year }\end{array}$ & $\begin{array}{l}\text { Mean: } 500,000 \\
\text { SD: } 50,000^{b}\end{array}$ & $N(500,000,50,000)$ & $x_{\# H D}$ \\
\hline Mutell et al ${ }^{15}$ & $\begin{array}{l}\text { Assumed reduction factor for } \\
\text { ESAs and IV iron associated } \\
\text { with ferric citrate usage }\end{array}$ & $\begin{array}{l}\text { ESAs: } 0.1,0.2,0.3 \\
\text { Fe: } 0.3,0.4,0.5\end{array}$ & $\begin{array}{l}\text { TRI }(0.05,0.15,0.1) \text {, } \\
\text { TRI }(0.15,0.25,0.2) \text {, } \\
\text { TRI }(0.25,0.35,0.3) \text {, } \\
\text { TRI }(0.35,0.45,0.4) \text {, } \\
\text { TRI }(0.45,0.55,0.5)\end{array}$ & $x_{\mathrm{RF}}, x_{\mathrm{RF} 2}$ \\
\hline $\begin{array}{l}\text { CMS base rate costs } \\
\text { PPPY }(2011)^{c}\end{array}$ & ESA PPPY costs & $\begin{array}{l}\left.\text { Lower (Aranesp }{ }^{\circledR}\right) \text { : US\$I,426 } \\
\left.\text { Middle (Epogen }{ }^{\circledR}\right) \text { : US } \$ 12,243 \\
\text { Upper: US\$13,467b }\end{array}$ & TRI $(I, 426,13,467,12,243)$ & $x_{\mathrm{ESA}}$ \\
\hline & IV iron PPPY costs & $\begin{array}{l}\left.\text { Lower (Ferrlecit }{ }^{\circledR}\right) \text { : US } \$ 432 \\
\text { Middle (Venofer }{ }^{\circledR} \text { ): US\$I,057 } \\
\text { Upper: US } \$ 1,162^{\text {b }}\end{array}$ & TRI $(432, \mathrm{I}, 162, \mathrm{I}, 057)$ & $x_{\mathrm{Fe}}$ \\
\hline $\begin{array}{l}\text { CMS actual utilization } \\
\text { (20II) PPPY costs }\end{array}$ & ESA PPPY costs & $\begin{array}{l}\text { Lower (Aranesp): US } \$ 543 \\
\text { Middle (Epogen): US\$9,545 } \\
\text { Upper: US\$10,499b }\end{array}$ & TRI $(543,10,499,9,545)$ & $x_{\mathrm{ESA}}$ \\
\hline & IV iron PPPY costs & $\begin{array}{l}\text { Lower (Ferrlecit): US\$209 } \\
\text { Middle (Venofer): US\$I, I } 48 \\
\text { Upper: US } \$ 1,263^{b}\end{array}$ & TRI $(209,1,263, I, \mid 48)$ & $x_{\mathrm{Fe}}$ \\
\hline
\end{tabular}

Table I Sources of uncertainty and parameter values assumed and probability distributions simulated

Notes: ${ }^{\mathrm{N}} \mathrm{N}(\mu, \sigma)=$ normal distribution with mean $\mu$, and standard deviation $\sigma$, and TRI $(\mathrm{a}, \mathrm{c}, \mathrm{b})=$ triangular distribution with lower bound a, upper bound $\mathrm{c}$, and mode b; bupper bound set to middle value plus 10\%; 'RED BOOK TM (Truven Health Analytics New York, NY, USA) values and CMS base rate and actual utilization of ESA and IV iron used to derive PPPY costs of ESA and Fe. Epogen ${ }^{\circledR}$ and AranesP ${ }^{\circledR}$ are manufactured by Amgen Inc., CA, USA; Venofer ${ }^{\circledR}$ is manufactured by American Regent, Inc., NY, USA; and Ferrlecit ${ }^{\circledR}$ is manufactured by Sanofi US, Bridgewater, NJ, USA.

Abbreviations: CMS, Centers for Medicare and Medicaid Services; ESA, erythropoietin-stimulating agent; HD, hemodialysis; IV, intravenous; PPPY, per-patient-per-year; SD, standard deviation; USRDS, United States Renal Data System. 
where $y_{\text {reduction }}$ is the projected annual cost reduction due to assumed fractional reduction arising from the use of ferric citrate. Assuming ferric citrate usage reduces ESA and IV iron required for therapy, we employed the costreduction model (Equation 2) using nine combinations of reduction values of $0.10,0.20$, and 0.30 for ESAs and 0.30 , 0.40 , and 0.50 for iron. These reduction values were midpoints (modes) of the triangle distributions listed in Table 1. The point estimate of the outcome for annual cost or annual cost reduction was taken to be the median value of $y$ with subjective confidence intervals based on the 20th and 80th percentiles. Adjusted annual costs were based on subtracting the cost reduction and its confidence limits from the total cost and its confidence interval. Monte Carlo analyses were run for CMS base rates and actual 2011 utilization of ESAs and IV iron reported by CMS. Two tables with nine combinations of reduction factors were generated for each source of costs (base rate and actual usage in 2011). A second set of tables was constructed containing the adjusted total annual costs based on subtracting the nine values of cost reductions from the single total annual cost for base rate and actual usage in 2011 and its subjective confidence intervals.

The effect of correlation between ESAs and IV iron was assessed for both the total cost and cost-reduction models assuming only the CMS base rate. Sensitivity analysis was performed for only the total cost model when ESA and IV iron input distributions were based on the CMS base rate. Sensitivity was determined by regressing all 15,000 realizations of output total cost on quantiles for ESA PPPY cost, IV iron PPPY cost, and number of patients undergoing dialysis per year, and by estimating the partial coefficient of determination for each input variable. The percentage of variance in total cost explained by each input variable is reflected by the partial coefficient of determination.

\section{Results}

Figures 1-4 show the Monte Carlo input uncertainty distributions for ESA and iron PPPY costs. Figure 1 illustrates the uncertainty distribution for PPPY ESA cost (USD) based on CMS base rate for which a triangular distribution was assumed, with a lower bound of US\$1,426, upper bound of US $\$ 13,467$, and mode of US\$12,243. Figure 2 shows the uncertainty distribution for PPPY IV iron cost based on the CMS base rate. A triangular distribution was assumed, with a lower bound of US\$432, upper bound of US\$1,162, and mode of US\$1,057. Figure 3 provides the uncertainty distribution for an example reduction factor based on a triangular distribution, having a lower bound of 0.05 , upper bound of 0.15 , and mode of 0.1 . Figure 4 shows the uncertainty distribution for the number of patients undergoing dialysis per year, assuming a normal distribution, with mean of 500,000 and standard deviation of 50,000. Figure

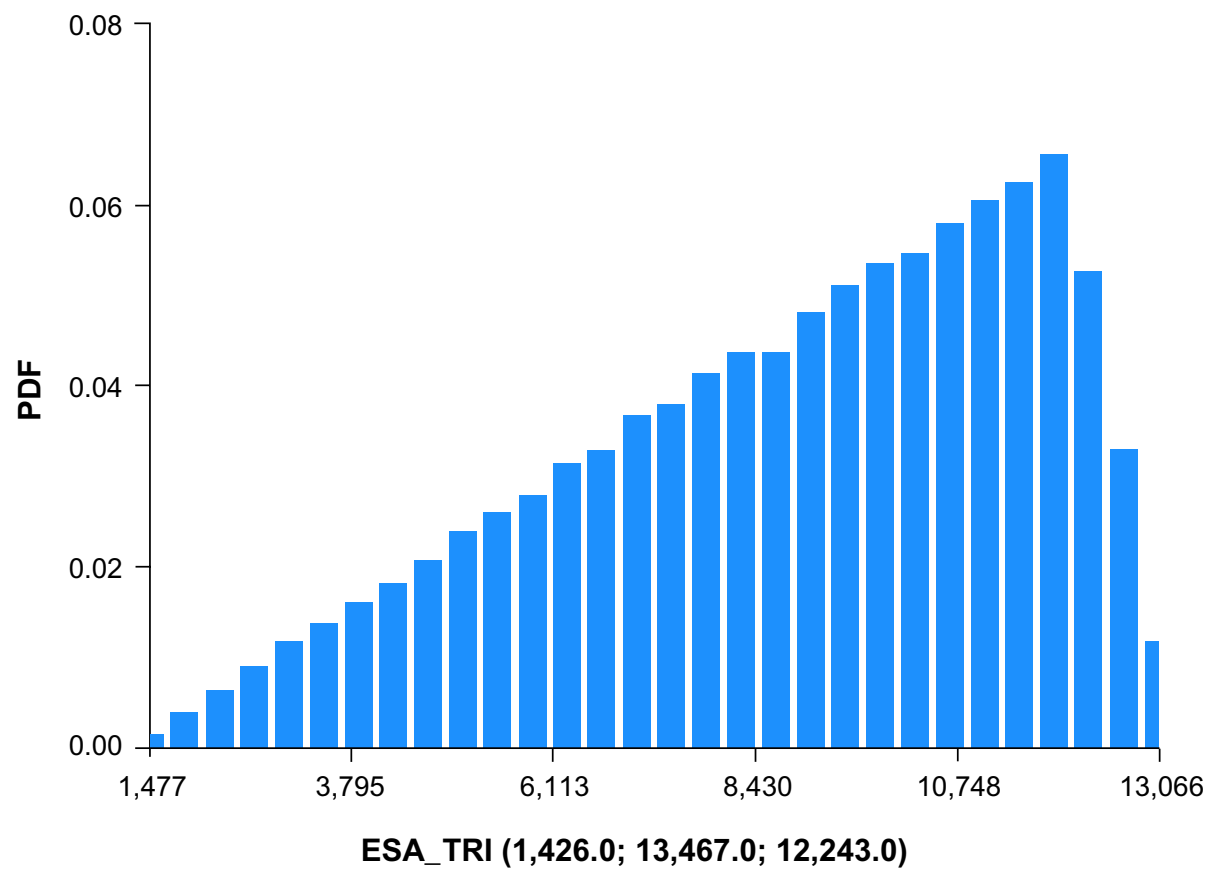

Figure I Uncertainty distribution for PPPY ESA cost (USD) based on CMS base rate.

Notes: Triangular distribution assumed, with lower bound of US $\$ 1,426$, upper bound of US $\$ 13,467$, and mode of US\$12,243. Distribution derived from I5,000 realizations. Abbreviations: CMS, Centers for Medicare and Medicaid Services; ESA, erythropoietin-stimulating agent; PPPY, per-patient-per-year; PDF, probability density function. 


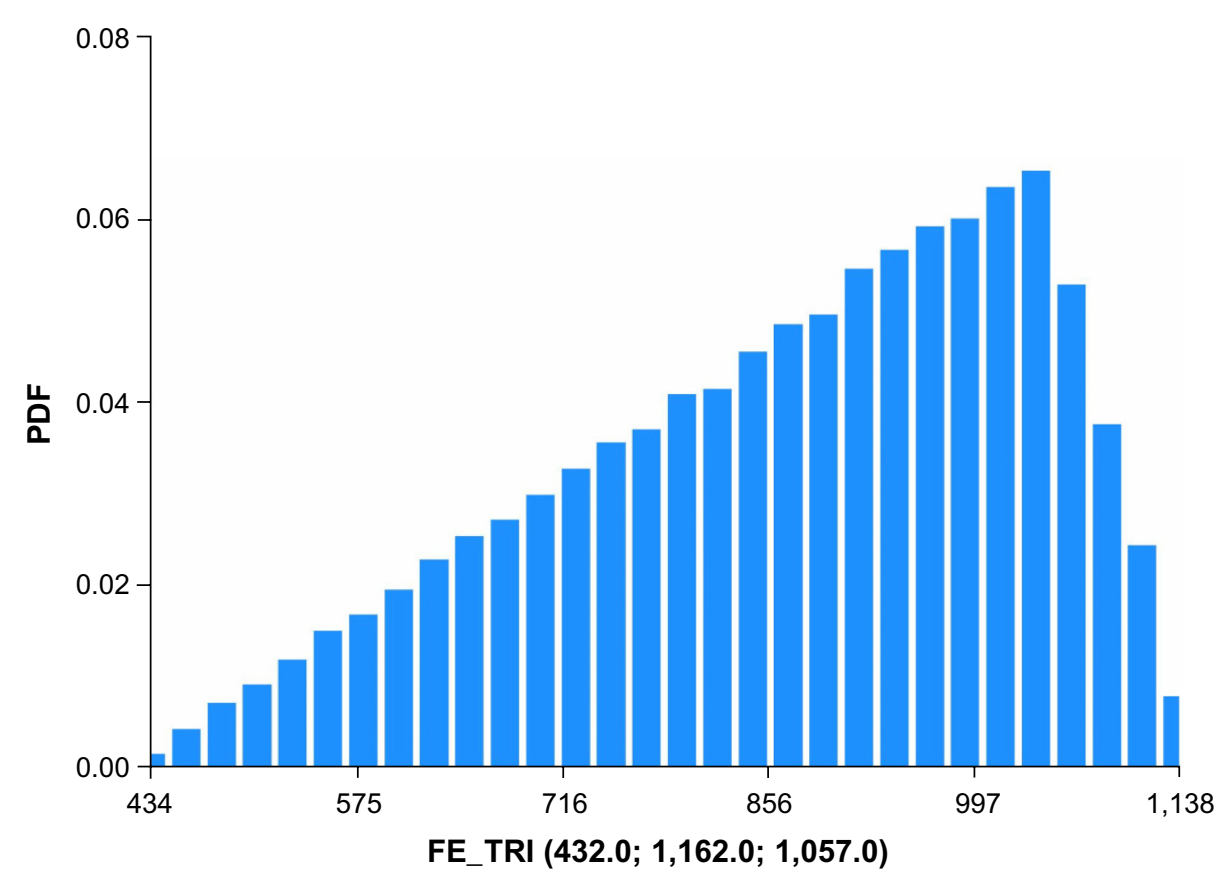

Figure 2 Uncertainty distribution for PPPY IV iron cost (USD) based on CMS base rate.

Notes: Triangular distribution assumed, with lower bound of US $\$ 432$, upper bound of US $\$ 1,162$, and mode of US $\$ 1,057$. Distribution derived from I5,000 realizations. Abbreviations: CMS, Centers for Medicare and Medicaid Services; FE, intravenous iron; PPPY, per-patient-per-year; PDF, probability density function.

5 reveals the outcome from Monte Carlo analysis showing the distribution of total cost (billion USD) of ESAs and IV iron assuming base rate values and 500,000 patients per year without applying reductions for ferric citrate usage. As Figure 5 illustrates, the annual total cost of ESAs and IV iron for dialysis patients, assuming the CMS base rate values, determined by Monte Carlo analysis was 5.127 (3.664-6.260) billion USD. For actual utilization in 2011, however, the annual total cost of ESAs and IV iron was 3.981 (2.780-4.930) billion USD (data not shown).

Table 2 lists the projected annual ferric citrate-based cost reductions for ESAs and IV iron, assuming CMS base

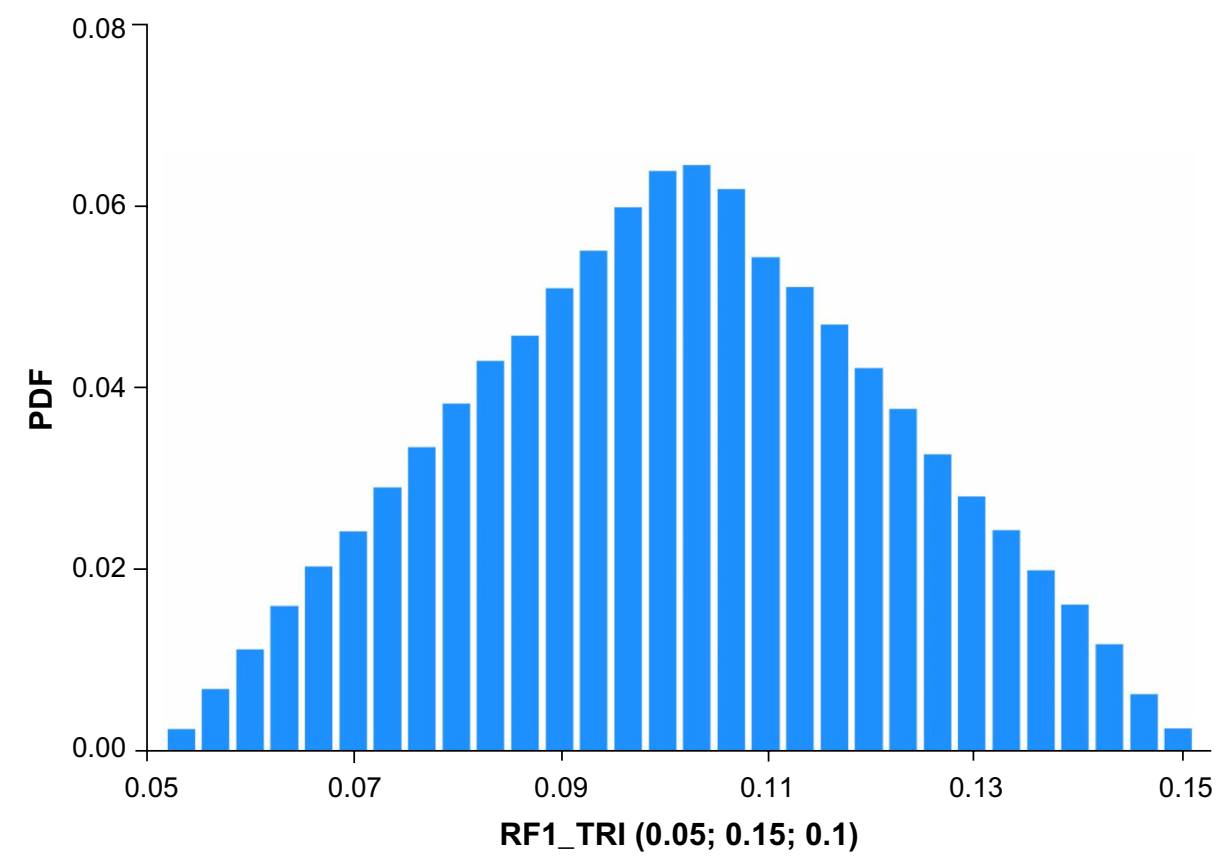

Figure 3 Example uncertainty distribution for a reduction factor for the cost-reduction model.

Notes: Triangular distribution assumed, with lower bound of 0.05 , upper bound of 0.15 , and mode of 0.1 . Distribution derived from I5,000 realizations. Abbreviation: PDF, probability density function. 


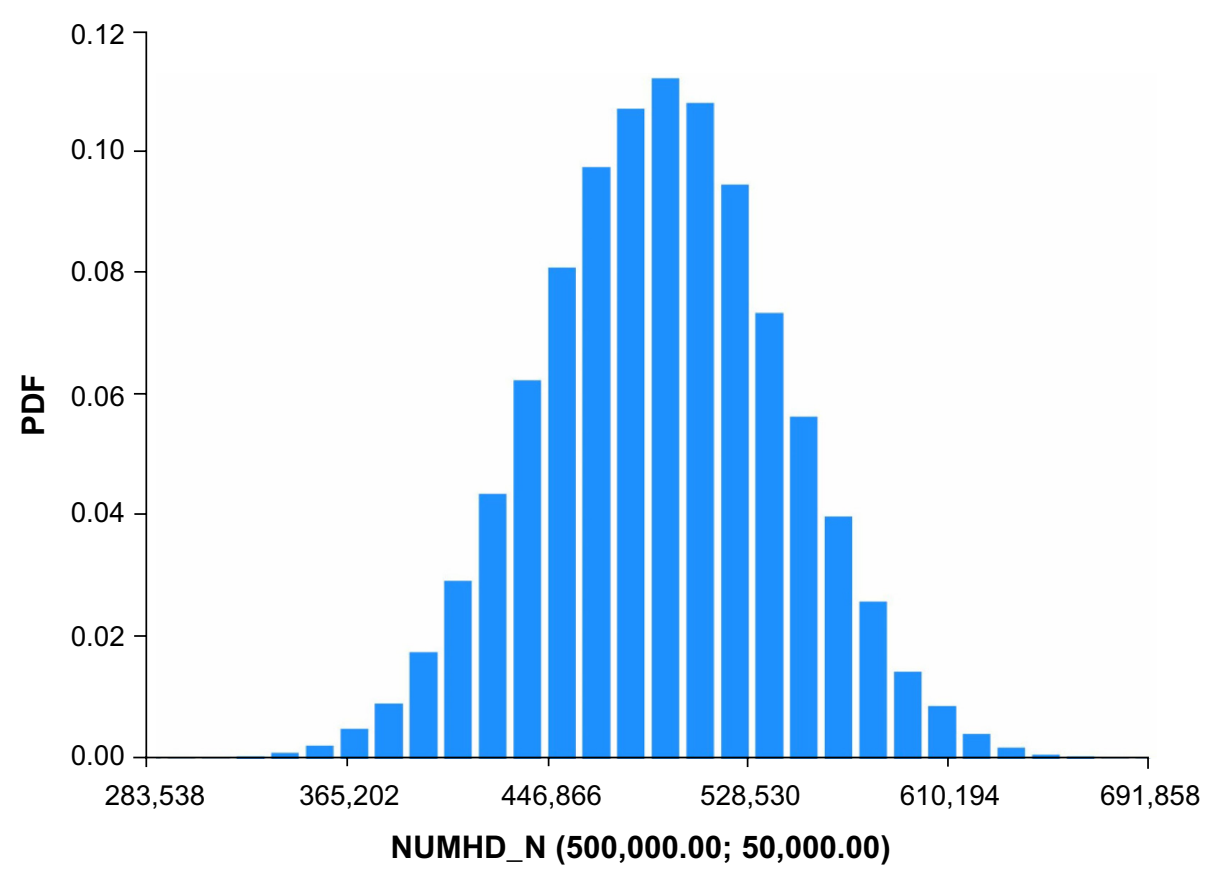

Figure 4 Uncertainty distribution for number of patients undergoing HD per year.

Notes: Normal distribution assumed, with mean of 500,000 and SD of 50,000. Distribution derived from I5,000 realizations.

Abbreviations: HD, hemodialysis; NUMHD, number of patients undergoing HD; SD, standard deviation; PDF, probability density function.

rates, which ranged from 0.578 to 1.604 billion USD. Under the CMS base rates, if ferric citrate usage resulted in a $20 \%$ reduction in ESA usage and 40\% reduction in IV iron usage, then the projected cost savings would be 1.089 (0.796-1.346) billion USD (Table 2). This would result in a total cost change from $5.127(3.664-6.260)$ to $4.038(2.868-4.914)$ billion USD, equal to $-21.2 \%$ (Table 3 ).

Table 4 lists ferric citrate-based projected cost reductions based on the 2011 actual utilization of ESAs and IV iron, for which the range was $0.467-1.273$ billion USD. If ferric

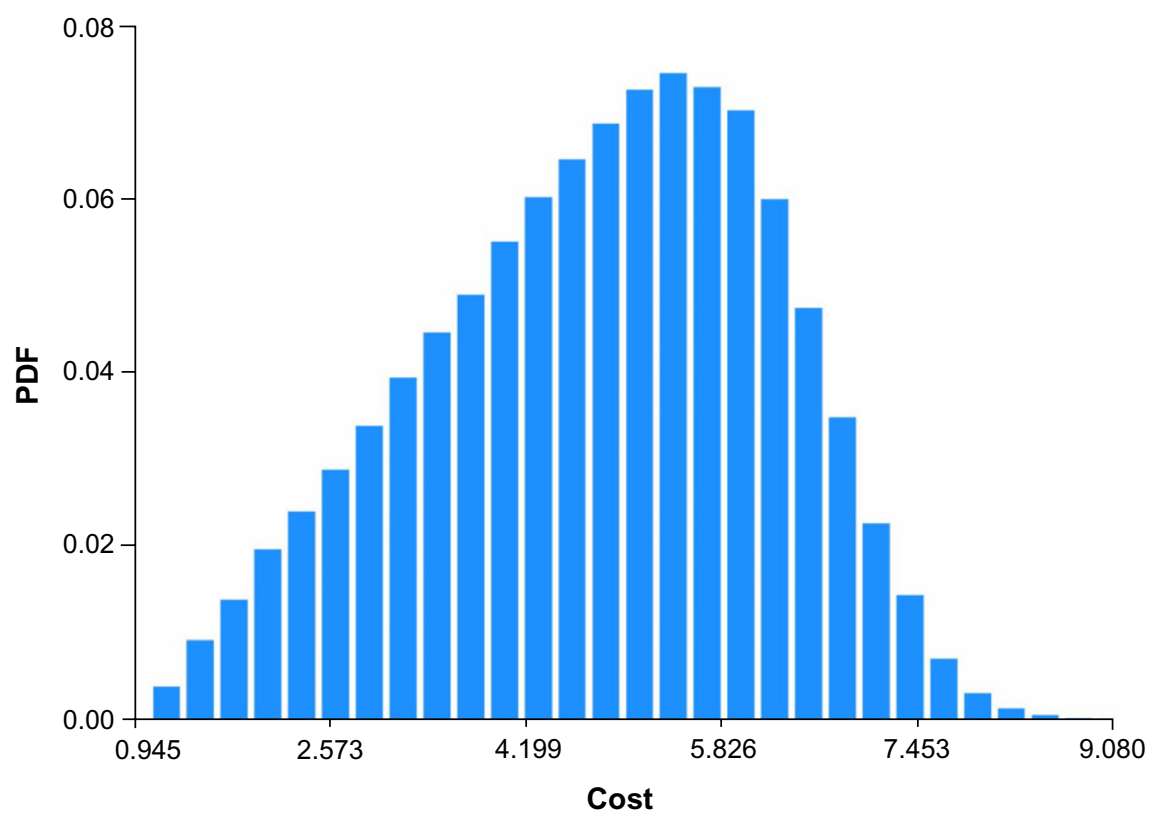

Figure 5 Output distribution for annual total cost (billion USD) of ESA and IV iron, assuming base rate values and 500,000 patients per year. Notes: Median value of distribution is 5.127 (3.664-6.260) billion USD. No reductions to ESA or IV iron costs were assumed for ferric citrate usage. Distribution derived from 15,000 realizations.

Abbreviations: ESA, erythropoietin-stimulating agent; IV, intravenous; PDF, probability density function. 
Table 2 Annual cost reductions based on base rate as a function of reduction in ESAs and IV iron arising from ferric citrate usage

\begin{tabular}{llll}
\hline ESA reduction & \multicolumn{3}{l}{ IV iron reduction } \\
\cline { 2 - 4 } & $\mathbf{0 . 3 0}$ & $\mathbf{0 . 4 0}$ & $\mathbf{0 . 5 0}$ \\
\hline 0.10 & 0.578 & 0.621 & 0.663 \\
& $(0.428-0.740)$ & $(0.470-0.786)$ & $(0.508-0.825)$ \\
0.20 & 1.047 & 1.089 & 1.137 \\
& $(0.762-1.307)$ & $(0.796-1.346)^{\mathrm{a}}$ & $(0.846-1.402)$ \\
0.30 & 1.522 & 1.560 & 1.604 \\
& $(1.082-1.883)$ & $(1.124-1.925)$ & $(1.165-1.973)$ \\
\hline
\end{tabular}

Notes: Cost reductions are expressed as median and 20th and 80th percentiles (in billion USD). aMost likely result.

Abbreviations: ESA, erythropoietin-stimulating agent; IV, intravenous.

citrate reduced ESA usage by $20 \%$ and IV iron usage by $40 \%$, then under the actual utilization costs, the projected annual cost reduction would be $0.868(0.632-1.084)$ billion USD (Table 4). The total annual cost based on actual utilization would then be reduced from $3.981(2.780-4.930)$ to 3.113 (2.148-3.845) billion USD, which equates to a $-21.8 \%$ change (Table 5).

The results described above assume no correlation between ESAs and IV iron. Figure 6 shows that when correlation between ESAs and IV iron is taken into consideration and ferric citrate assumingly reduces ESA utilization by $20 \%$ and IV iron use by $40 \%$, the quartiles of total cost and cost reduction increase slightly with increasing correlation in the range -1 to 1 . Analogously, Figures 7 and 8 illustrate the standard deviation, skewness, and kurtosis of total cost and cost reduction, assuming ferric citrate reduces ESA requirements by $20 \%$ and IV iron usage by $40 \%$.

Lastly, Figure 9 shows the sensitivity of total cost (Equation 1) to ESAs, IV iron, and number of patients undergoing dialysis, assuming only the CMS base rate in Table 1 and no correlation between ESAs and IV iron. The percentage variance explanation of total cost by ESA, number of patients

Table 3 Annual cost of 5.127 (3.664-6.260) billion USD adjusted for reductions

\begin{tabular}{llll}
\hline ESA reduction & \multicolumn{3}{l}{ IV iron reduction } \\
\cline { 2 - 4 } & $\mathbf{0 . 3 0}$ & $\mathbf{0 . 4 0}$ & $\mathbf{0 . 5 0}$ \\
\hline 0.10 & 4.549 & 4.506 & 4.464 \\
& $(3.236-5.520)$ & $(3.194-5.475)$ & $(3.156-5.435)$ \\
0.20 & 4.080 & 4.038 & 3.990 \\
& $(2.902-4.953)$ & $(2.868-4.914)^{\mathrm{a}}$ & $(2.818-4.859)$ \\
0.30 & 3.605 & 3.567 & 3.523 \\
& $(2.582-4.377)$ & $(2.539-4.335)$ & $(2.498-4.288)$ \\
\hline
\end{tabular}

Notes: Costs are expressed as the median and 20th and 80th percentiles (in billion

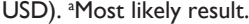

Abbreviations: ESA, erythropoietin-stimulating agent; IV, intravenous.
Table 4 Annual cost reductions based on 20 II actual utilization of ESA and IV iron as a function of reduction in ESA and IV iron arising from ferric citrate usage

\begin{tabular}{llll}
\hline ESA reduction & \multicolumn{3}{l}{ IV iron reduction } \\
\cline { 2 - 4 } & $\mathbf{0 . 3 0}$ & $\mathbf{0 . 4 0}$ & $\mathbf{0 . 5 0}$ \\
\hline 0.10 & 0.467 & $0.5 I I$ & 0.555 \\
& $(0.341-0.600)$ & $(0.382-0.649)$ & $(0.423-0.692)$ \\
0.20 & 0.830 & 0.868 & 0.918 \\
& $(0.584-1.035)$ & $(0.632-1.084)^{\mathrm{a}}$ & $(0.672-1.132)$ \\
0.30 & 1.192 & 1.236 & 1.273 \\
& $(0.831-1.486)$ & $(0.869-1.529)$ & $(0.915-1.578)$ \\
\hline
\end{tabular}

Notes: Cost reductions are expressed as the median and 20th and 80th percentiles (in billion USD). aMost likely result.

Abbreviations: ESA, erythropoietin-stimulating agent; IV, intravenous.

undergoing dialysis per year, and IV iron were 99\%, 93.3\%, and $24.8 \%$, respectively.

\section{Discussion}

The physiological effects of ferric citrate are multiple in that it reduces serum phosphorus levels while simultaneously increasing transferrin saturation. Previous literature has reported a reduction in ESA dose requirements as a result of increased iron stores. ${ }^{16}$ In our investigation, we determined that cost reductions for ESAs and IV iron as a result of using ferric citrate as a phosphate binder suggest potentially large national cost savings. The cost-offset analysis by Mutell et al supports our findings of potential savings attributed to reduced ESA and IV iron usage. ${ }^{15}$

The CMS reported that actual utilization in 2011 was lower than the base rate and will likely result in future reductions in the base rate. ${ }^{2}$ A potential study bias may be that this would likely reduce overall costs of ESA and iron CMS reimbursement. We also only considered the four anemia-management drugs Epogen, Aranesp, Venofer, and Ferrlecit, which were the only drugs reimbursed under the CMS bundled prospective payment system that applied to the entire US. Our results

Table 5 Annual cost of 3.98 I (2.780-4.930) billion USD adjusted for reductions

\begin{tabular}{llll}
\hline ESA reduction & \multicolumn{3}{l}{ IV iron reduction } \\
\cline { 2 - 4 } & $\mathbf{0 . 3 0}$ & $\mathbf{0 . 4 0}$ & $\mathbf{0 . 5 0}$ \\
\hline 0.10 & 3.514 & 3.470 & 3.425 \\
& $(2.440-4.330)$ & $(2.398-4.280)$ & $(2.357-4.238)$ \\
0.20 & 3.151 & 3.113 & 3.063 \\
& $(2.197-3.895)$ & $(2.148-3.845)^{\mathrm{a}}$ & $(2.108-3.797)$ \\
0.30 & 2.789 & 2.745 & 2.708 \\
& $(1.950-3.443)$ & $(1.911-3.400)$ & $(1.866-3.352)$ \\
\hline
\end{tabular}

Notes: Costs are expressed as the median and 20th and 80 th percentiles (in billion USD). a Most likely result.

Abbreviations: ESA, erythropoietin-stimulating agent; IV, intravenous. 


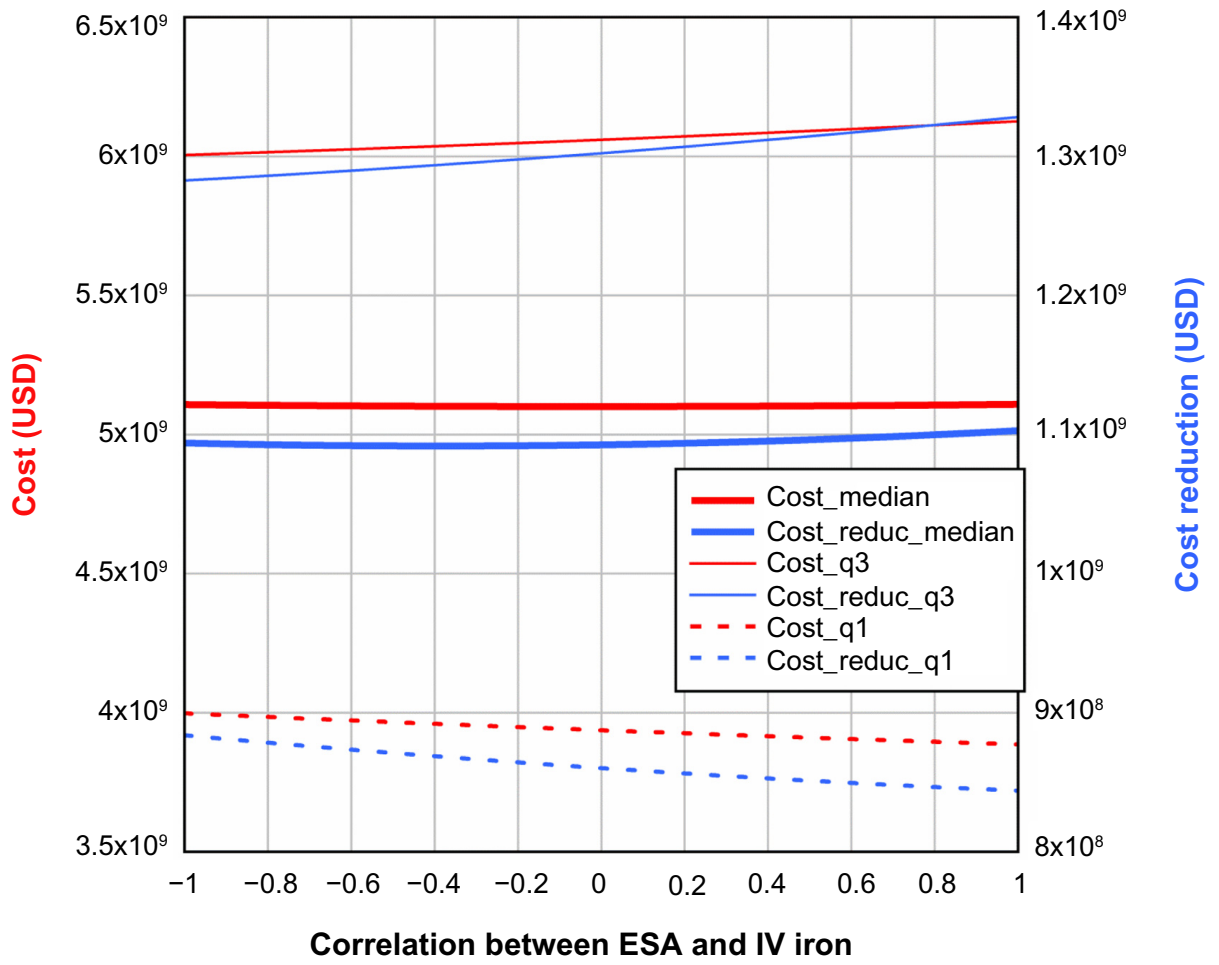

Figure 6 Total cost and cost reduction (USD) as a function of correlation between ESA and IV iron.

Note: For the cost-reduction model, it was assumed that ferric citrate reduced ESA usage by $20 \%$ and IV iron usage by $40 \%$.

Abbreviations: ESA, erythropoietin-stimulating agent; IV, intravenous; qI, 25th percentile, q3, 75th percentile.

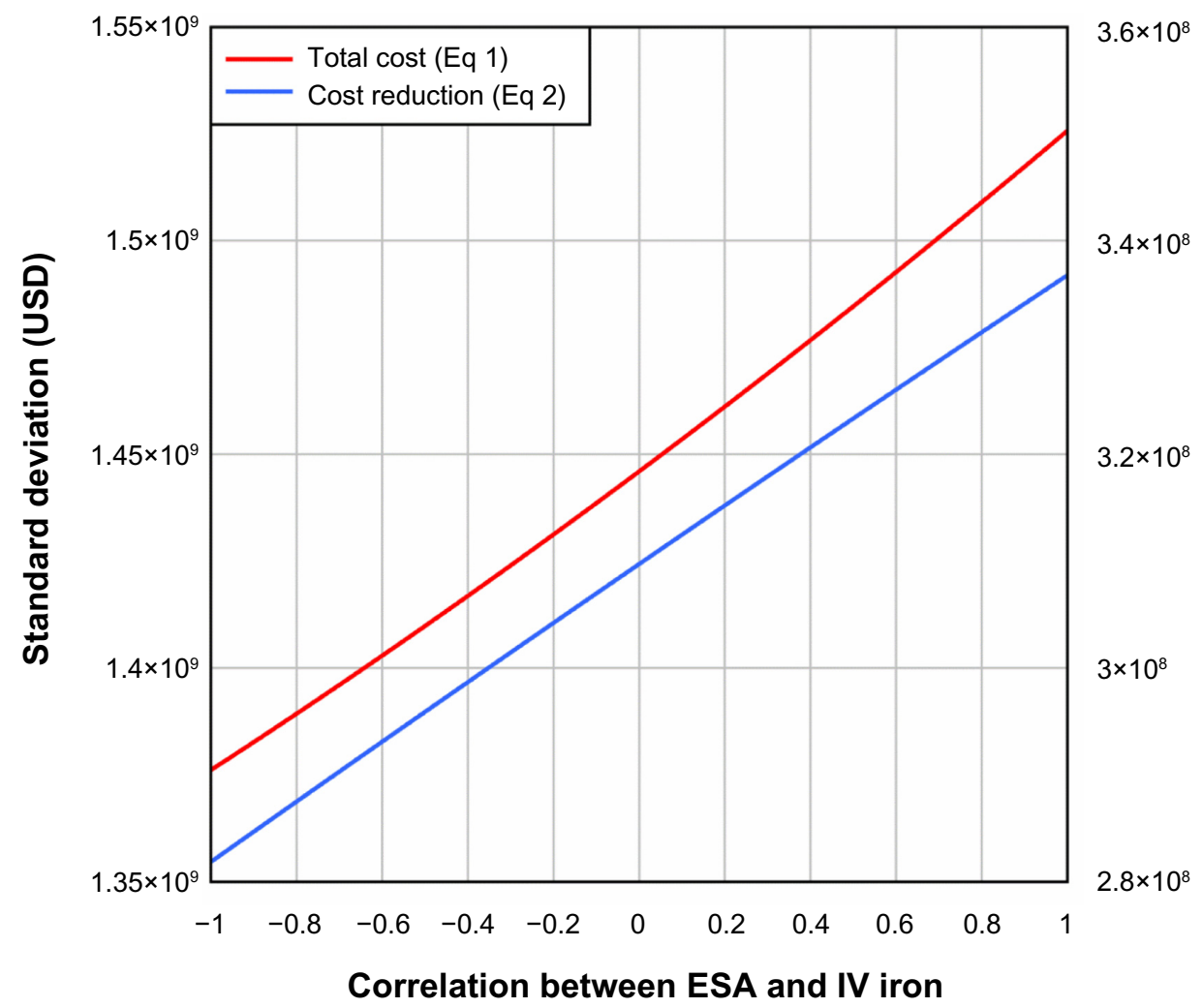

Figure 7 Standard deviation of total cost and cost reduction (USD) as a function of correlation between ESA and IV iron.

Note: For the cost-reduction model, it was assumed that ferric citrate reduced ESA usage by $20 \%$ and IV iron usage by $40 \%$.

Abbreviations: ESA, erythropoietin-stimulating agent; IV, intravenous. 


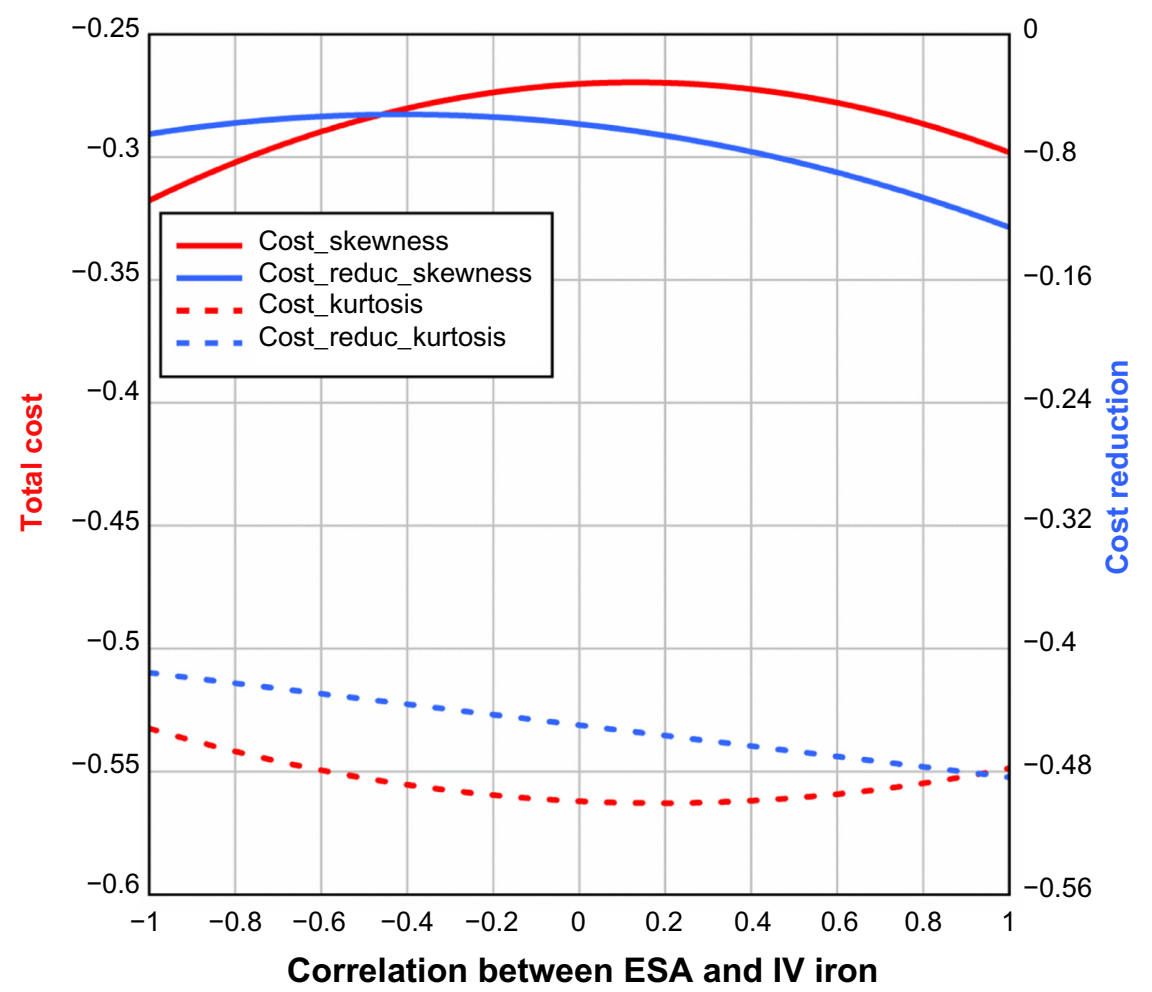

Figure 8 Skewness and kurtosis of total cost and cost reduction (USD) as a function of correlation between ESA and IV iron. Note: For the cost-reduction model, it was assumed that ferric citrate reduced ESA usage by $20 \%$ and IV iron usage by $40 \%$.

Abbreviations: ESA, erythropoietin-stimulating agent; IV, intravenous.

would naturally not apply to institutions with contract pricing and different PPPY costs. Our model is applicable to large-scale outpatient dialysis facilities. Nevertheless, if our assumptions hold, we believe that our results should reliably project reductions in ESA and iron utilization cost savings

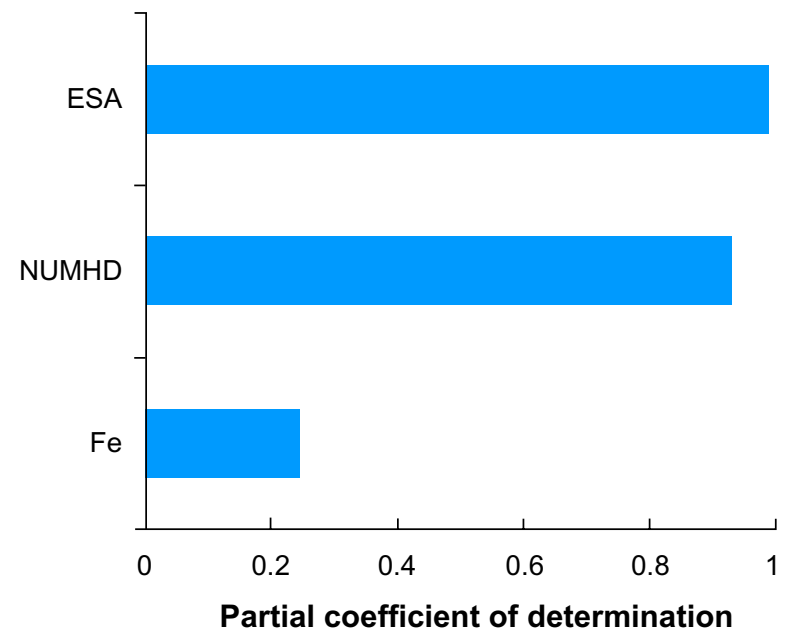

Figure 9 Sensitivity of total cost model to input values when ESA PPPY and IV iron PPPY costs were based on the CMS base rate.

Note: Zero correlation between ESA and IV iron assumed.

Abbreviations: CMS, Centers for Medicare and Medicaid Services; ESA, erythropoietin-stimulating agent; Fe, intravenous iron; NUMHD, number of patients undergoing hemodialysis; PPPY, per-patient-per-year. for a majority of anemia-management programs in ESRD. Ferric citrate is not yet approved for use in the US, so there are no national statistics for dosage and reimbursement, or cost history. Hence, our cost-reduction model is based on the likely range of percentage reduction in ESA and IV iron usage associated with ferric citrate usage. There are presently no large-scale Phase III or IV investigations quantifying the reduction in ESA dose as a result of ferric citrate use. Studies are also needed to determine the efficacy of ferric citrate in improving anemia via the oral route in patients with significant iron deficiency or long-standing iron deficiency.

Our implementation of Monte Carlo analysis has the advantage of providing the uncertainty distribution of the final outcome costs. It is inappropriate to multiply central mean values of factors together to determine the sum product of independent factors for cost-benefit analysis because no information is provided regarding distributional properties of cost and associated confidence intervals. Monte Carlo analysis generates thousands of realizations based on random draws from input probability distributions, which are input into the model equation during each iteration. This approach builds a subjective empirical probability distribution for the model's outcome, along with the uncertainty from which lower and upper confidence intervals are obtained. 
We used the median of each outcome distribution as the central estimate of cost, and the 20th percentile for the lower bound and the 80th percentile for the upper bound of the confidence limit.

It is well known that dependence between input factors affects the width of the outcome uncertainty during Monte Carlo analysis. By varying correlation between ESAs and IV iron, we observed that the standard deviation, skewness, and kurtosis of model outcome were affected more than the median and confidence intervals, so our results corroborate the correlation-width assumption. Because we were not attempting to model the dose-response relationship between ESAs and IV iron, we provide a combination of reductions in the usage of ESAs and IV iron arising from ferric citrate usage, so that the reader can identify an estimate of the likely cost reduction. Overall, it was observed that the medians of cost and cost reduction were not strongly influenced by ESA-IV iron correlation.

The sensitivity analysis results suggest that the majority of variance in total cost depends mostly on ESA PPPY and the number of patients undergoing dialysis per year. IV iron PPPY costs had a much lower contribution to the variance of total cost. Sensitivity is based on the squared partial correlation between outcome total costs and each individual input factor, assuming the other inputs are held constant. The additional post hoc evaluation regarding sensitivity reveals another advantage of Monte Carlo analysis, since straightforward use of mean values to determine cost savings is much less informative. Future studies are needed to further quantify the benefit of ferric citrate usage in terms of cost reduction of anemia management for patients with ESRD.

\section{Conclusion}

It is likely that US health care costs for anemia-management drugs associated with ESRD among dialysis patients can be reduced by using ferric citrate as a phosphate binder. At present, however, FDA approval of ferric citrate is pending (target date June 7, 2014, http://www.keryx.com), and therefore, there are no Phase IV post-marketing data to confirm cost-savings of ESAs and IV iron during ESRD anemia management as a result of using ferric citrate. In the absence of Phase IV post-marketing data, we performed Monte Carlo uncertainty analysis to gain insight into possible cost reductions arising from use of ferric citrate. Our results indicate that, if ferric citrate usage results in a $20 \%$ reduction in ESA usage and 40\% reduction in IV iron usage, then $0.9-1.1$ billion USD can be saved by using ferric citrate as a phosphate binder among ESRD patients.

\section{Acknowledgments}

We would like to acknowledge Drs Juan J Olivero Sr and Juan J Olivero Jr for their helpful discussions on the ESRD bundled prospective payment system and the place in therapy of ferric citrate.

\section{Disclosure}

Authors declare no conflicts of interest in this work.

\section{References}

1. United States Renal Data System, USRDS. 2013 Annual Data Report: Atlas of Chronic Kidney Disease and End-Stage Renal Disease in the United States. Bethesda, MD: National Institutes of Health, National Institute of Diabetes and Digestive and Kidney Diseases; 2013.

2. US Department of Health and Human Services, Office of the Inspector General. Medicare and Beneficiaries Could Save Millions if Dialysis Payments were Adjusted for Anemia Management Drug Utilization (A-01-12-00522). May 2013.

3. US Government Accountability Office. Medicare: High-Expenditure Part B Drugs (GAO-13-46R Medicare Part B Drug Spending). October 12, 2012.

4. Weiss G, Goodnough L. Anemia of chronic disease. $N$ Engl J Med. 2005;352:1011-1023.

5. Singh A, Szczech L, Tang K, et al. Correction of anemia with epoetin alfa in chronic kidney disease. $N$ Engl J Med. 2006;355: 2085-2098.

6. Drüeke T, Locatelli F, Clyne N, et al. Normalization of hemoglobin level in patients with chronic kidney disease and anemia. $N$ Engl J Med. 2006;355:2071-2084.

7. Pfeffer M, Burdmann E, Chen C, et al. A trial of darbepoetin alfa in type 2 diabetes and chronic kidney disease. $N$ Engl $J$ Med. 2009;361:2019-2032.

8. Horl WH. Clinical aspects of iron use in the anemia of kidney disease. J Am Soc Nephrol. 2007;18:382-393.

9. KDIGO. Clinical Practice Guideline for Anemia in Chronic Kidney Disease. Kidney Int Suppl. 2012;2:4(2).

10. Cupisti A, Gallieni M, Rizzo MA, et al. Phosphate Control in Dialysis. Int J Nephrol Renovasc Dis. 2013;6:193-205.

11. Savica V, Calo L, Monardo P, et al. Phosphate binders and management of hyperphosphatemia in end-stage renal disease. Nephrol Dial Transpl. 2006;21:2065-2068.

12. Yokoyama K, Hirakata H, Akiba T, et al. Effect of oral JTT-751 (ferric citrate) on hyperphosphatemia in hemodialysis patients: results of a randomized, double-blind, placebo-controlled trial. Am J Nephrol. 2012;36(5):478-487.

13. Bond C, Jensen D, Wang S, et al. A meta-analysis of ferric citrate for hyperphosphatemia: the effects of an oral iron-containing phosphate binder on serum ferritin and saturated transferrin in hemodialysis patients. Paper presented at: 49th ERA-EDTA Congress 2012 (European Renal Association - European Dialysis and Transplant Association); May 24-27, 2012; Paris, France.

14. Poradosu E, Rubin J, Bond C, et al. TSAT and serum ferritin increases associated with ferric citrate use may lead to dialysis cost savings. Abstract presented at: 43rd ANNA National Symposium (American Nephrology Nurses' Association); April 29-May 2, 2012; Orlando, Florida.

15. Mutell R, Rubin J, Bond T, et al. Reduced use of erythropoiesisstimulating agents and intravenous iron with ferric citrate: a managed care cost-offset model. Int J Nephrol Renovasc Dis. 2013;6: 79-87.

16. Harmankaya O, Eran A. Low-dose intravenous iron administration in chronic hemodialysis patients treated with recombinant human erythroopoietin. Renal Failure. 2002;24(2):245-247. 
International Journal of Nephrology and Renovascular Disease

\section{Publish your work in this journal}

The International Journal of Nephrology and Renovascular Disease is an international, peer-reviewed open-access journal focusing on the pathophysiology of the kidney and vascular supply. Epidemiology, screening, diagnosis, and treatment interventions are covered as well as basic science, biochemical and immunological studies. The journal welcomes

original research, clinical studies, reviews \& evaluations, expert opinion and commentary, case reports and extended reports. The manuscript management system is completely online and includes a very quick and fair peerreview system, which is all easy to use. Visit http://www.dovepress.com/ testimonials.php to read real quotes from published authors.

Submit your manuscript here: http://www.dovepress.com/international-journal-of-nephrology-and-renovascular-disease-journal 